\title{
Formação em Avaliação Psicológica: uma análise das disciplinas
}

\author{
Ana Paula Porto Noronha \\ Universidade São Francisco
}

\begin{abstract}
RESUMO
O presente estudo objetivou investigar os conteúdos e disciplinas relacionadas à avaliação psicológica a partir da análise de 41 ementas de universidades brasileiras, sendo $71,7 \%$ privadas e 28,3\% públicas. As ementas foram consultadas nos sites das instituições de ensino superior. Os resultados revelaram que há uma nomenclatura variada para as disciplinas; que 78\% das ementas são de disciplinas oferecidas até o $4^{\circ}$ semestre; e que os conteúdos prioritariamente trabalhados são técnicas de avaliação e aplicação, mensuração psicológica e técnicas projetivas. Sugere-se que novos estudos sejam desenvolvidos, com a ampliação do número de ementas consultadas e com outros critérios de análise, a fim de se promover maior reflexão e discussão a respeito da formação em avaliação psicológica.
\end{abstract}

Palavras-chave: avaliação psicológica; testes psicológicos; ensino de testes

\begin{abstract}
Psychological Assessment Training: an analysis of courses

The present study aimed to investigate the contents and the courses related to psychological assessment by analyzing the course syllabus of 41 Brazilian universities, $71,7 \%$ were private institutions and $28,3 \%$ were public universities. The course syllabus were assessed through the website of each institution. The results have shown a varied nomenclature for the psychological assessment courses, with $78 \%$ of the courses being offered until the fourth semester. The main contents addressed were assessment techniques and application, psychological measurement and projective techniques. New studies with a larger number of syllabus and other analysis criteria are suggested in order to promote a better understanding and discussion about the undergraduate courses of psychological assessment.
\end{abstract}

Keywords: psychological assessment; psychological tests; test training

Recentemente no Brasil foram aprovadas novas diretrizes curriculares para os cursos de graduação em Psicologia, cuja organização foi pautada na definição de competências e habilidades profissionais. A proposta teve como objetivo orientar o planejamento, a implementação e a avaliação dos cursos de psicologia e, nesse sentido, foram definidos os seguintes eixos estruturantes, a saber, fundamentos epistemológicos e históricos; fenômenos e processos psicológicos básicos; aspectos metodológicos; procedimentos para a investigação científica e a prática profissional; conhecimento de campos afins e práticas profissionais (Conselho Federal de Psicologia, 2003).

De acordo com Bastos (2002) as diretrizes enfatizam um núcleo comum de formação que, por sua vez, tem a função de assegurar uma formação básica dentre os vários estados brasileiros. Os "perfis de formação" seriam as características específicas entre os diversos cursos e estariam centrados na vocação ou demandas sociais, regionais e locais. Além disso, o aluno teria mais possibilidades de fazer escolhas relacionadas à sua formação, por meio das ênfases curriculares, ou seja, espaços de aprofundamentos teórico-práticos.

No que se refere especialmente ao eixo "procedimentos para a investigação científica e a prática profissional", das novas diretrizes, no qual se localiza a avaliação psicológica, objeto de estudo do presente trabalho, vale destacar que ele foi entendido como a busca da compreensão aprofundada dos fenômenos e processos psicológicos no campo da psicologia científica (CFP, 2003). Ainda, foram consideradas competências do psicólogo, a escolha e a utilização de instrumentos e procedimentos de coleta de dados, considerando os problemas quanto ao uso, assim como a construção e os parâmetros psicométricos; além da avaliação cognitiva, comportamental e afetiva em diferentes contextos. 
Dentre os eixos estruturantes, em torno dos quais os conteúdos curriculares devem se articular, Yamamoto (2000) destaca que nos procedimentos para investigação científica e prática profissional, deve estar incluído o domínio técnico imprescindível para o uso de instrumentos de avaliação e de intervenção, assim como a competência para construir, avaliar e adequar instrumentos a contextos dos diferentes campos profissionais da psicologia.

De alguma forma, essas questões sobre a formação vêm sendo discutidas, desde que a profissão foi reconhecida como tal, reforçando a tese de que há muito se procura a melhor forma de se preparar o psicólogo para desenvolver com eficiência suas atividades profissionais (Duran, 1994; Maciel, 1994; Noronha, 2002; Sbardelini, 1991). Nesse sentido, é pertinente a colocação de Maciel (1994), ao afirmar que o aumento do número de cursos de graduação nem sempre foi acompanhado da crescente qualidade deles.

O Conselho Federal de Psicologia, juntamente como outros órgãos e associações científicas, vem discutindo e propondo fóruns de reflexão sobre a formação em psicologia, tais como o Encontro Nacional com gestores de cursos de Psicologia de todo o país, ocorrido em 1992, o II Congresso Nacional de Psicologia (1996), cujo eixo temático foi formação em Psicologia ou ainda, o I Encontro Nacional da Associação Brasileira de Ensino de Psicologia (ABEP) em 2000, dentre outros (CFP, 2003). Embora os eventos tenham sido distintos, em comum eles revelaram a preocupação com a preparação profissional.

Apesar de se ter uma definição simples e clara do que se pretende objetivar com a formação específica na área de avaliação psicológica brasileira, parece que a grande dificuldade tem sido traduzir tais premissas em ações mais concretas, do tipo, como formar, o que priorizar e qual a melhor metodologia a ser empregada nesse processo. É fato que esses questionamentos não são recentes na literatura brasileira, já que é possível encontrar artigos como o de Sbardelini (1991) que retrata a problematização antes destacada. No entanto, apesar da discussão ser antiga, parece que as soluções para os problemas advindas de formações insatisfatórias estão bastante presentes na realidade dos psicólogos.

Ao serem questionados sobre os problemas mais graves e mais freqüentes na avaliação psicológica, 214 sujeitos responderam que em ambas as categorias encontra-se a formação profissional (Noronha, 2002). No estudo de Pereira (1972), dentre outras considerações, constatou-se que os psicólogos não possuem uma imagem adequada das suas funções sociais, que é reduzida a preocupação com a pesquisa, e que há desinteresse dos cursos e dos profissionais por algumas áreas de aplicação da psicologia. Almeida, Prieto, Muñiz e Bartram (1998) constataram entre psicólogos portugueses, espanhóis e de países ibero-americanos que existe uma clara relação entre problemas nos instrumentos psicológicos e problemas na formação profissional do psicólogo que constrói e que usa os referidos instrumentos.

Também nessa direção, qual seja, a de analisar a formação em avaliação psicológica, Noronha, Baldo, Barbin e Freitas (2003) investigaram o conhecimento de 180 estudantes de psicologia, de $1^{\circ}$ e $5^{\circ}$ anos, a respeito de conteúdos relacionados à avaliação psicológica. Os resultados indicaram que houve diferença significativa entre os grupos de $1^{\circ}$ e $5^{\circ}$ anos em aproximadamente metade dos itens avaliados e que, de uma forma geral, os sujeitos do quinto ano obtiveram melhores desempenhos do que os do primeiro, embora em algumas questões o índice de acerto dos primeiros anistas tenha sido superior.

A formação em avaliação psicológica também foi tema de pesquisa de Noronha, Baldo, Almeida, Freitas, Barbin e Cozoli (2004). As autoras fizeram um estudo comparativo entre estudantes de psicologia e de engenharia em relação a um questionário contendo 35 itens dicotômicos, abordando questões sobre o conceito de avaliação psicológica, aprendizagem e uso de instrumentos. Os resultados revelaram que o grupo de psicologia apresentou melhor desempenho que o grupo de engenharia em grande parte das questões, tendo havido diferença significativa entre os grupos na maioria deles.

Alves, Alchieri e Marques (2002) consultaram professores de avaliação psicológica, com o intuito de mapear os testes ensinados e quais conteúdos deveriam ser considerados básicos para uma boa atuação profissional. Foram recebidos 184 questionários, de 172 professores, de 62 cursos diferentes. Os resultados indicaram que o número médio e a variedade de testes ensinados é maior do que o que professores consideram como conteúdo básico ou mínimo, e que há semelhança entre os testes mais ensinados e os indicados como básicos.

Nada obstante, não parece ser possível desvincular todo o processo de desenvolvimento da avaliação psicológica e, em especial, dos testes psicológicos, da formação na área. Sob esta perspectiva, Pasquali e Alchieri (2001) estabelecem cinco períodos da evolu- 
ção científica no país, sendo que o primeiro (18361930), trouxe um pequeno volume de contribuições, embora importantes, como a criação do primeiro laboratório de Psicologia no Hospital Nacional de Alienados e o uso da Escala Binet-Simon. O segundo período (1930-1962) já é mais marcante e se resume pelas inaugurações de centros de pesquisa, tais como o do ISOP, o do SENAI e do CETEPP. No período que compreende de 1962 a 1970 (terceiro) houve a oficialização da psicologia como profissão, além do reconhecimento do curso de psicologia e da abertura da avaliação psicológica como atividade profissional. De 1970 a 1990 houve o incremento do ensino e da pesquisa psicológica, enquanto que a partir daí, o $5^{\circ}$ período preocupou-se com a epistemologia dos instrumentos de medida.

Especialmente no que se refere à avaliação da formação do psicólogo, Landeira-Fernandez e Primi (2000) estudaram o Exame Nacional de Cursos (ENC) de Psicologia, realizado por todos os formandos no ano de 2000. Os resultados revelaram que participantes provenientes de diferentes universidades apresentaram desempenhos altamente diferenciados e proporcionais aos conceitos dos cursos. Ainda, uma análise dos conteúdos específicos de cada um dos 39 itens do ENC 2000 revelou uma deficiência na formação acadêmica dos formandos nas áreas relacionadas aos processos psicológicos básicos, à metodologia científica e às medidas e técnicas de avaliação psicológica.

Como se vê, embora a psicologia brasileira esteja em franco desenvolvimento (Hutz, McCarthy \& Gomes, 2003), assim como a avaliação psicológica (Primi, 2003), ainda serão necessários muitos investimentos para que se consiga um arsenal teórico e metodológico brasileiros que subsidie toda a ação do psicólogo, ou grande parte dela. Parte dos investimentos refere-se a uma formação profissional consistente na área de avaliação, de tal sorte que os psicólogos consigam atuar de maneira coerente nos mais diversos contextos profissionais.

Ao lado disso, devem ser estabelecidos conteúdos, assim como devem ser discutidas estratégias de ensino. Muitos estudos trataram do tema "formação", com enfoques diferentes (alunos, docentes, instrumentos mais ensinados, dentre outros), mas poucos se enveredaram por questões relacionadas aos assuntos ou instrumentos que devem compor a formação.

Destaque deve ser dado ao trabalho de Noronha e cols. (2002), que sugeriu que os seguintes temas façam parte dos currículos das disciplinas de avaliação, a saber, teoria da medida e psicometria; avaliação da inteligência e da personalidade; e prática de planejamento, execução e redação de resultados. Com o intuito de fornecer parâmetros para as ações futuras no que concerne à formação em avaliação psicológica, Hutz e Bandeira (2003), sugeriram que haja o estabelecimento de conteúdos básicos a serem contemplados na graduação; que se desenvolvam estratégias para a educação continuada e especialização; que se criem programas para a qualificação de professores; que haja apoio à criação, ao desenvolvimento e à integração dos laboratórios de avaliação, assim como a comunicação constante e eficiente entre pesquisadores, professores, estudantes e profissionais que utilizam a avaliação psicológica, dentre outras. Tendo em vista o exposto, o presente trabalho visou investigar os conteúdos e as disciplinas relacionadas à avaliação psicológica, de diferentes universidades brasileiras, por meio da análise das ementas.

\section{MÉTODO}

\section{Material}

Foram utilizadas 41 ementas de disciplinas de avaliação psicológica de 38 cursos de Psicologia brasileiros, que possuíam sites e disponibilizavam o programa das disciplinas. As análises foram referentes a instituições de ensino superior, sendo $71,7 \%$ particulares e $28,3 \%$ públicas. No que se refere à distribuição das instituições pelas regiões do país, vale destacar que o Sudeste esteve mais representado com $47,9 \%$ das instituições; $25 \%$ das universidades eram da região Sul; $16,7 \%$ do Nordeste; $8,4 \%$ do Centro-Oeste e $2,0 \%$ do Norte.

\section{Procedimento e critérios de análise das ementas}

Realizou-se uma busca na Internet, em todos os sites disponíveis, das Instituições de Ensino Superior que oferecessem cursos de Psicologia. Considerando os objetivos desse estudo, montou-se uma planilha eletrônica na qual eram incorporadas além da descrição das instituições, as disciplinas ofertadas referentes à avaliação psicológica, os respectivos semestres e a carga horária delas. Vale destacar que foram consultadas apenas as ementas e os programas que se encontravam on-line. Após isso, procedeu-se à análise, cujos resultados são apresentados a seguir.

A fim de se investigar os conteúdos tratados, cada ementa foi dividida em unidades de análise, conforme os preceitos de Bardin (1971). 
1. Teoria Psicológica: reuniu as respostas que abordavam os conceitos pertinentes às características da mensuração e da medida em Psicologia, bem como limitações e dificuldades da medida em Ciências Humanas, além de construtos psicológi$\cos$ não relacionados a nenhum instrumento em especial.

2. Testes de Personalidade - conceitos, aplicação e avaliação: os argumentos foram incluídos nesta categoria de resposta quando estavam relacionados aos testes de personalidade, à fundamentação, às instruções de aplicação, avaliação e interpretação.

3. Testes de inteligência - conceitos, aplicação e avaliação: os argumentos foram incluídos nesta categoria de resposta quando estavam relacionados aos testes de inteligência, à fundamentação, às instruções de aplicação, avaliação e interpretação.

4. Técnicas de Avaliação e de aplicação de testes: envolveu os argumentos que tratam de técnicas de avaliação (observação, entrevista, hora de jogo, dentre outros) e de aplicação de testes (não relacionado a nenhum instrumento específico).

5. Psicometria: reuniu as respostas que abordavam a padronização, normalização, validade, precisão e princípios estatísticos.

6. Elaboração de laudos: conteúdos relacionados à elaboração de laudos diagnósticos e relatórios.

7. Cuidados éticos na Avaliação Psicológica: aborda as respostas relacionadas aos princípios éticos e deontológicos da Avaliação Psicológica.

8. Avaliação Psicológica - características, processos e fundamentos: estão incluídas as respostas que tratam da Avaliação Psicológica e dos elementos acima discriminados em diferentes contextos profissionais.

9. Técnicas Projetivas: foram incluídas nesta categoria, as respostas relacionadas aos testes de inteligência, à fundamentação, às instruções de aplicação, avaliação e interpretação.

10. Psicodiagnóstico: as respostas pertinentes a esta categoria foram as que abordavam o "processo psicodiagnóstico", com a inclusão de testes e técnicas de avaliação.

11. Outros testes.

As unidades de análise foram definidas pela autora. $\mathrm{O}$ enquadramento nas categorias contou com o auxílio de dois mestrandos em psicologia, sendo que a decisão final, no caso de dúvida, foi consensual.

\section{RESULTADOS E DISCUSSÃO}

Os resultados foram organizados em dois grupos, sendo que o primeiro destinou-se a discutir questões descritivas referentes às disciplinas, enquanto o outro, analisou os conteúdos tratados nas ementas e programas. Inicialmente chamou a atenção a diversidade encontrada em relação a número de disciplinas e momentos do curso nos quais são ministradas. Nada obstante, tal condição plural tem sido evidenciada por vários pesquisadores brasileiros (Almeida, Prieto, Muñiz \& Bartram, 1998; Noronha, 2002; Noronha e cols., 2005).

No tocante à nomenclatura, foram encontradas, Técnicas do Exame Psicológico 1, Técnicas do Exame Psicológico 2, Técnicas do Exame Psicológico 3, Psicometria, Avaliação Psicológica 1, Avaliação Psicológica 2, Avaliação Psicológica 3, Inventários de Personalidade, Técnicas Projetivas, Prática de Avaliação Psicológica, Técnicas de Avaliação Psicológica, dentre outras denominações. Os diferentes nomes atribuídos às disciplinas que abordam conteúdos de avaliação psicológica podem evidenciar uma ausência de coerência e lógica, que traduz a falta de articulação da própria área. Embora esta análise seja inicial, permite identificar a dificuldade na concordância de nomenclatura das disciplinas entre as universidades distribuídas pelos Estados brasileiros, como já apontado pelo trabalho de Alchieri e Bandeira (2002).

No que se refere ao momento de início das disciplinas de avaliação psicológica no Curso de Psicologia, observou-se que $78 \%$ dos cursos oferecem a primeira disciplina até o $4^{\circ}$ semestre, ou seja, ainda nos dois anos iniciais da formação, embora em algumas universidades o primeiro contato com a avaliação psicológica seja posterior. A Tabela 1 apresenta essas informações. 
Tabela 1. Série inicial das disciplinas de avaliação psicológica

\begin{tabular}{ccc}
\hline Semestres & Freqüência & $\%$ \\
\hline $1^{\circ}$ & 5 & 12,2 \\
$2^{\circ}$ & 8 & 19,5 \\
$3^{\circ}$ & 12 & 29,3 \\
$4^{\circ}$ & 7 & 17,1 \\
$5^{\circ}$ & 6 & 14,6 \\
$6^{\circ}$ & 1 & 2,4 \\
$7^{\circ}$ & 2 & 4,9 \\
\hline Total & 41 & 100 \\
\hline
\end{tabular}

O fato das disciplinas serem ministradas, em sua maioria, até o $4^{\circ}$ semestre, pode sugerir que elas se localizam dentre o leque de conteúdos básicos, o que contraria as considerações de Sbardelini (1991), Noronha e cols. (2002) e Hutz e Bandeira (2003), no sentido de que teoria da medida e psicometria; avaliação da inteligência e da personalidade; e prática de planejamento, execução e redação de resultados não sejam abordadas no período inicial da formação, uma vez que o aluno já deveria ter alguns domínios para melhor aproveitar a informação.

A carga horária dos Cursos de Psicologia mostrouse bastante variada, de tal modo que a média foi de 4.320 horas ( $\mathrm{DP}=607,672)$, com um mínimo de 3.240 e máximo de 5.724 horas. No que diz respeito à carga horária específica das disciplinas relacionadas à avaliação psicológica, encontrou-se uma média de 226,61 horas, com a mínima de 68 e a máxima de 570 horas $(\mathrm{DP}=108,359)$. O dado está relacionado ao número de disciplinas, e nessa direção, encontrou-se que a área de avaliação psicológica tem sido contemplada com uma média de 3,29 disciplinas, variando de 1 a 7 $(\mathrm{DP}=1,55)$, o que está de acordo com os achados de Alves, Alchieri e Marques (2002). Embora não se esperasse consenso entre as universidades e respectivos cursos, considerando a diversidade necessária, em direção oposta está o cenário brasileiro, qual seja o permeado por falta de critérios balizadores, que garantam uma formação satisfatória.

A Tabela 2 apresenta as freqüências das unidades de análise acima descritas. Os resultados revelaram que técnicas de avaliação e aplicação, mensuração psicológica e técnicas projetivas são as categorias mais presentes nas ementas analisadas. Em contrapartida, a psicometria, os testes de inteligência e outros testes são menos representados. Tais dados podem ser corroborados pelo estudo de Noronha e cols. (2005), no sentido de que as técnicas projetivas são as mais ensinadas. Em contrapartida, as de inteligência diferentemente do presente estudo, também apareceram como dentre as mais discutidas.

Tabela 2. Freqüência e porcentagem das unidades de análise

\begin{tabular}{lcc}
\hline Categorias & Freqüência & $\%$ \\
\hline Técnicas de Avaliação & 23 & 56,1 \\
Mensuração Psicológica & 20 & 48,8 \\
Técnicas Projetivas & 19 & 46,3 \\
Psicodiagnóstico & 17 & 41,5 \\
Testes de Personalidade & 12 & 29,3 \\
Cuidados éticos & 12 & 29,3 \\
Avaliação Psicológica & 11 & 26,8 \\
Elaboração de laudos & 11 & 26,8 \\
Testes de inteligência & 9 & 22,0 \\
Psicometria & 8 & 19,5 \\
Outros testes & 5 & 12,2 \\
\hline
\end{tabular}

A justificativa para a diferença de resultados entre as pesquisas se dá em razão de algumas possibilidades. As categorias utilizadas nos estudos são diferen- tes; Noronha e cols. (2005) fizeram uso de 16 elementos de análise, enquanto o estudo atual, definiu 11; utilizou-se nesse trabalho um universo maior de 
universidades; e, possivelmente a mais relevante das reflexões centre-se no fato de que a maior inserção de conteúdos relacionados à mensuração psicológica se dê como resultado de um processo de revisão da área de avaliação psicológica, no qual as discussões em pequenos fóruns, em eventos científicos, as pesquisas, assim como o maior envolvimento de docentes e pesquisadores estejam gerando modificações.

Embora essa visão seja positiva, não é possível deixar de destacar as impropriedades encontradas. Nessa direção, o ensino ainda é predominantemente voltado à avaliação da personalidade e da inteligência, ficando outros construtos psicológicos minimizados em importância quando da realização de avaliações psicológicas.

Para a análise da divisão das unidades de análise nas disciplinas, estas últimas foram agrupadas em razão do nome, ou seja, todas as disciplinas de Avaliação Psicológica foram reunidas, mesmo possuindo a nomenclatura Avaliação psicológica I, II ou III. A apresentação dessa informação se dá na Tabela 3, e sob esta perspectiva, os blocos de disciplinas abordam, em média, de 3 (Psicodiagnóstico) a 4,8 (Psicometria) conteúdos.

\section{Tabela 3. Unidades de análise por disciplina}

\begin{tabular}{|c|c|c|c|c|c|c|c|c|c|c|c|c|c|}
\hline & 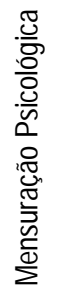 & 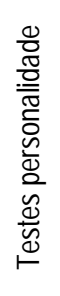 & 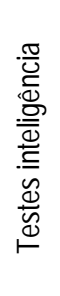 & 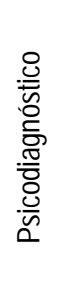 & 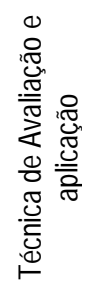 & 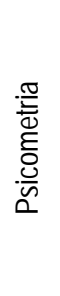 & 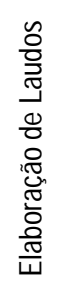 & 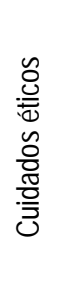 & 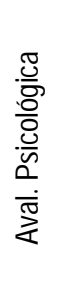 & 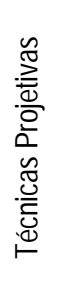 & 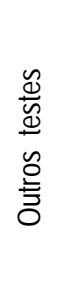 & 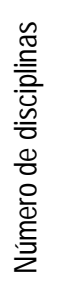 & 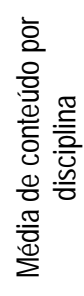 \\
\hline Avaliação Psicológica & 2 & 2 & 2 & 1 & 3 & 1 & 1 & 2 & 1 & 2 & & 5 & 3,4 \\
\hline Inventário de Personalidade & & 1 & & & 1 & & & & & 1 & & 1 & 3 \\
\hline PAP & 1 & 1 & 1 & 1 & 1 & & 3 & 1 & 2 & & 1 & 3 & 4 \\
\hline Psicodiagnóstico & 1 & 1 & & & 1 & & & & & & & 1 & 3 \\
\hline Psicometria & 4 & 1 & 1 & 1 & 3 & 3 & 1 & 1 & 2 & 1 & 1 & 4 & 4,8 \\
\hline Teste Projetivo & 1 & 2 & 1 & 1 & 3 & & 1 & 3 & & 4 & 1 & 5 & 3,4 \\
\hline TAP & 2 & 1 & 1 & 3 & 3 & 1 & 2 & 1 & 2 & 5 & & 6 & 3,5 \\
\hline TEAP & & 1 & 1 & 3 & 1 & 1 & 2 & 1 & & & 1 & 3 & 3,7 \\
\hline TEP & 9 & 2 & 2 & 7 & 7 & 2 & 1 & 3 & 4 & 6 & 1 & 13 & 3,4 \\
\hline Total & 20 & 12 & 9 & 17 & 23 & 8 & 11 & 12 & 11 & 19 & 5 & 41 & \\
\hline
\end{tabular}

Uma busca mais detalhada pelos conteúdos tratados nos diferentes blocos de disciplinas retrata um cenário bastante confuso, à medida que não existe especificidade de conteúdo para algumas disciplinas, como seria o caso de Teste Projetivo, na qual são trabalhados temas diversos, como mensuração psicológica, testes de personalidade, testes de inteligência, psicodiagnóstico, técnicas de avaliação e aplicação, elaboração de laudos, cuidados éticos, técnicas projetivas e outros testes. Ao lado disso, Psicometria e Técnicas de Exame Psicológico abordam todos os conteúdos.

Assim, é possível supor que as ementas das instituições analisadas são bastante distintas, ou seja, disciplinas com o mesmo nome não tratam do mesmo conteúdo. Também nessa direção cabe ressaltar que as unidades de análise previstas para esse estudo poderiam compor uma única disciplina de avaliação psicológica, considerando a complexidade do tema e volume de literatura disponível. Em contrapartida, o que se vê, em geral, é uma reunião de temas, o que tende a prejudicar a qualidade do ensino e da preparação profissional.

\section{CONSIDERAÇÕES FINAIS}

Embora a formação em avaliação psicológica venha sendo repensada, ainda serão necessários muitos investimentos de docentes e pesquisadoras no sentido da melhoria da qualidade. Acredita-se, como já enfatizado por autores diversos, que a atuação competente está relacionada à formação sólida. Tal premissa é válida também para a área de avaliação psicológica, à 
medida que práticas profissionais incompetentes têm sido sistematicamente veiculadas nos meios de divulgação oficial da profissão.

A preparação do psicólogo no Brasil tem sido mais técnica, de tal forma que se ensina a "aplicar", mas não necessariamente a se refletir sobre as razões dessa ou de outra aplicação qualquer. Assim tem sido na avaliação. $\mathrm{O}$ ensino desvinculado da sólida formação teórica; um número de disciplinas razoável, que aborda muitos conteúdos, possivelmente em uma carga horária insatisfatória, com a intenção de se ensinar um pouco de cada tema, tal como já previsto por Alves, Alchieri e Marques (2002).

Poder-se-ia perguntar qual a conseqüência decorrente desse quadro. É possível interpretar que o resultado não poderia ser diferente; psicólogos muitas vezes sem domínio técnico imprescindível para o uso de instrumentos de avaliação e de intervenção; psicólogos algumas vezes sem a competência para construir, avaliar e adequar instrumentos a contextos dos diferentes campos profissionais (Yamamoto, 2000); questionamentos a respeito de como formar, o que priorizar e qual a metodologia melhor a ser empregada neste processo (Sbardelini, 1991); reduzida preocupação com a pesquisa, e desinteresse dos cursos e dos profissionais por algumas áreas de aplicação da psicologia (Pereira, 1972; Landeira-Fernandez \& Primi, 2000).

Mais do que oferecer sugestões, esse estudo reforça outras teses já defendidas, no sentido de que a mudança é necessária. Outros aspectos merecem também investigação e sob esta perspectiva, outras universidades poderiam ser pesquisadas, deveria haver ampliação das ementas analisadas, bem como a consulta aos programas das disciplinas traria outras informações relevantes. Assim, o presente estudo não esgotou as possibilidades de pesquisa, embora possa suscitar reflexões para os interessados na área.

\section{REFERÊNCIAS}

Alchieri, J. C. \& Bandeira, D. R. (2002). Ensino da Avaliação Psicológica na Brasil. Em R. Primi (Org.), Temas em Avaliação Psicológica (p.35-39). Campinas: Impressão Digital do Brasil Gráfica e Editora Ltda.

Alves, I. C. A. B., Alchieri, J. C. \& Marques, K. C. (2002). As técnicas de exame psicológico ensinadas nos cursos de graduação de acordo com os professores. Psico USF, 7 (1):77-88.

Almeida, L. S., Prieto, G., Muñiz, J. \& Bartram, D. (1998). O uso dos testes em Portugal, Espanha e Países Iberoamericanos. Psychologica, 20, 41-55.

Bardin, L. (1977). Análise de conteúdo. Lisboa: Edições.
Bastos, A. V. B. (2002). Perfis de formação e ênfases curriculares: o que são e por que surgiram? Revista do Departamento de Psicologia da UFF, 14 (1), 31-58.

Conselho Federal de psicologia (2003). Diretrizes Curriculares para os Cursos de Graduação em Psicologia: propostas e análise. Brasília: Conselho Federal de Psicologia.

Duran, A. P. (1994). Alguns Dilemas na Formação do Psicólogo: buscando sugestões para superá-los. Em CFP (Org.), Psicólogo Brasileiro: práticas emergentes e desafios para a formação (pp. 45-53). São Paulo: Casa do Psicólogo.

Hutz, C. S. \& Bandeira, D. R. (2003). Avaliação Psicológica no Brasil: situação atual e desafios para o futuro. Em M. O. H. Yamamoto \& V. V. Gouveia (Orgs.), Construindo a psicologia brasileira: desafios da ciência e prática psicológica (pp. 261275). São Paulo: Casa do Psicólogo.

Hutz, C. S., McCarthy, S. \& Gomes, W. (2003). Psychology in Brazil: the road behind and the road ahead. Em M. J. Stevens \& D. Wedding (Eds.). Handbook of International Psychology (pp. 151-168). New York: Brunner-Routledge.

Maciel, R. (1994). Formação Banalizada. Em Conselho Regional de Psicologia. Uma Profissão Chamada Psicologia. São Paulo: Conselho Regional de Psicologia/06 ${ }^{\mathrm{a}}$ Região.

Landeira-Fernandez, J. \& Primi, R. (2002). Comparação do desempenho entre calouros e formandos no Provão de Psicologia 2000. Psicologia Reflexão e Crítica, 15 (1), 219-234.

Noronha, A. P. P. (2002). Os Problemas Mais Graves e Mais Freqüientes no Uso dos Testes Psicológicos. Psicologia: Reflexão e Crítica, 15 (1), 135-142.

Noronha, A. P. P., Ziviani, C., Hutz, C. S., Bandeira, D. R., Custódio, E. M., Alves, I. B., Alchieri, J. C., Borges, L. O., Pasquali, L., Primi, R. \& Domingues, S. F. (2002). Em defesa da avaliação psicológica. Avaliação Psicológica, 1 (1), 173174.

Noronha, A. P. P., Batista, M. A., Carvalho, L., Cobero, C., Cunha, N. B., Dell’Aglia, B. A. V., Filizatti, R., Zenorini, R. P. C. \& Santos, M. M. (2005). Ensino de Avaliação Psicológica em Instituições de Ensino Superior Brasileiras. Revista Universitas Ciências da Saúde, 3 (1), 1-14.

Noronha, A. P. P., Baldo, C. R., Barbin, P. F. \& Freitas, J. V. (2003). Conhecimento em avaliação psicológica: um estudo com alunos de psicologia. Psicologia Teoria e Prática, 5 (2), 37-46.

Noronha, A. P. P., Baldo, C. R., Almeida, M. C., Freitas, J. V., Barbin, P. F. \& Cozoli, J. (2004). Conhecimento de estudantes a respeito de conceitos de avaliação psicológica Psicologia em Estudo, 9 (2), 263-269.

Pasquali, L. \& Alchieri, J. C. (2001). Os Testes Psicológicos no Brasil. Em L. Pasquali (Org.), Técnicas de Exame Psicológico - TEP - manual (pp. 195-221). São Paulo: Casa do Psicólogo, Conselho Federal de Psicologia.

Pereira, S. L. M. (1972). As atividades profissionais do psicólogo em São Paulo. Tese de Doutorado Não-Publicada, Programa de PósGraduação em Psicologia Escolar e do Desenvolvimento, Universidade de São Paulo, São Paulo.

Primi, R. (Org.) (2003). Temas em Avaliação Psicológica. Campinas: Impressão Digital do Brasil Gráfica e Editora Ltda.

Sbardelini, E. T. B. (1991). Os mitos que envolvem os testes psicológicos. Documenta CRP-08, 1, 1, 53-57. 
Yamamoto, O. H. (2000). A LDB e a Psicologia. Psicologia Ciên-

\section{Sobre a autora:}

Ana Paula Porto Noronha: Doutora em Psicologia Ciência e Profissão pela Pontifícia Universidade Católica de Campinas. Docente do Programa de Pós-graduação Stricto Sensu em Psicologia da Universidade São Francisco. Bolsista Produtividade em Pesquisa do CNPq.

Endereço para correspondência: Rua Alexandre Rodrigues Barbosa, 45, Centro - 13251-900 Itatiba - SP - Endereço eletrônico: ana.noronha@saofrancisco.edu.br - ana.noronha@ pesquisador.cnpq.br 